\title{
External damping losses in measuring the vibration damping properties in lightly damped specimens using transient time-domain methods
}

Corresponding author:

Author 1: Joachim Vanwalleghem

Affiliation: Mechanics of Materials and Structures, Department of Materials Science and Engineering, Ghent University, Technologiepark-Zwijnaarde 903, 9052 Zwijnaarde, Belgium

Phone: +32 (0)93310420

Fax: +32 (0)9 2645833

E-mail: Joachim.Vanwalleghem@UGent.be

Author 2: Ives De Baere

Affiliation: Mechanics of Materials and Structures, Department of Materials Science and Engineering, Ghent University, Technologiepark-Zwijnaarde 903, 9052 Zwijnaarde, Belgium

Phone: +32 (0)93310420

Fax: +32 (0)9 2645833

E-mail: Ives.DeBaere@UGent.be

Author 3: Mia Loccufier

Affiliation: Department of Electrical Energy, Systems and Automation, Ghent University, Technologiepark-Zwijnaarde 914, 9052 Zwijnaarde, Belgium

Phone: +32 (0)9 2645587

Fax: +32 (0)9 2643582

E-mail: Mia.Loccufier@,UGent.be

Author 4: Wim Van Paepegem

Affiliation: Mechanics of Materials and Structures, Department of Materials Science and Engineering, Ghent University, Technologiepark-Zwijnaarde 903, 9052 Zwijnaarde, Belgium

Phone: +32 (0)93310432

Fax: +32 (0)9 2645833

E-mail: Wim.VanPaepegem@UGent.be 


\begin{abstract}
The contaminating effect of external damping sources to the overall measured damping of mechanical structures has always been an issue. Although those sources are qualitatively known, they are often not considered if damping properties are experimentally determined, yielding erroneous results. The aim of this paper is to quantify some of these undesired effects on the overall measured damping value. Free vibrations of steel plate specimens are used to list up several causes of external damping sources. As small modifications to the test setup may lead to totally different results, appropriate actions are offered to design a more accurate test setup. Known observations, such as the damping value's dependence on the specimen size and the excitation level are confirmed. Finally, it is shown that the damping capacity of one type of steel alloy can usually not be generalised to other steel alloys.
\end{abstract}

Keywords: material damping, experimental, measurement errors

\title{
1 Introduction
}

Vibration damping is inherently present in all structures. It represents the ability of the structure to attenuate structural vibrations. Unlike the mass and the stiffness, the damping is not straightforward to characterize as it stems from many sources: fluidstructure interaction, internal material damping and joint damping at interfaces $[1,2,3,4,5,6]$. Material damping properties have to be determined through experiments, hereby it is important to realize that other dissipation components also take part in the total energy dissipation. The global energy dissipation is referred as system damping; it includes all dissipation components. The inherent material damping is related to the internal stress-strain state which is represented by a material model. The corresponding parameters for the material model have to be determined through experiments conducted on coupon samples. This implies that results are then only applicable for the 
environment (including stress, strain, temperature, frequency, etc.) at which the experiments were conducted. Conclusions as to material damping are only meaningful if extraneous damping present during tests is eliminated or accounted for.

This is why Adams and Fox [12] reported many inconsistencies for damping values of nominally identical materials. This resulted from an inadequate knowledge of the distribution of energy loss in all parts of the apparatus, including that in the specimen itself. Even if one joint is the only significant source of energy dissipation, it may be sufficient to cause misleading results. Other issues affecting the damping capacity of the structure under test may be due to contact making (measurement) devices with as these can change its dynamics and definitely sway its damping $[13,14]$. Other measurement errors are related to data acquisition hardware: measurement noise or phase error by the instrumentation can lead to inaccurate damping results [15]. Although it is generally accepted that small changes to the measurement configuration may affect the damping results, especially when lightly damped specimens are tested, this is rarely quantified in terms of an effective damping contribution and consequently erroneous damping results are obtained.

This paper meets the missing knowledge in the material damping assessment techniques through an identification of external damping sources by means of transient timedomain methods. This measuring technique is part of the experimental modal analysis (EMA) test methodology; it focuses on measuring the damping capacity of the specimen when vibrating at a resonance frequency. Four influences which cause undesired damping are examined in detail: (i) the specimen excitation method, (ii) the specimen vibration response measurement, (iii) the air damping contribution and (iv) the boundary conditions, i.e. how the specimen is connected with the environment. These parameters all contribute to the global measured damping value. The aim of this 
research is to eliminate all contaminating contributions as much as possible and consequently reducing the initial system damping value to a final best estimate of the material damping value. The influence of external damping sources on the overall measured damping value will be expressed in terms of relative errors. As a result, external, unwanted, damping losses are quantified and a test setup which is less susceptible to additional damping effects is designed.

\section{Materials and methods}

\subsection{Theoretical damping modelling considerations}

Fitting a model onto the measured data is necessary for the experimental quantification of the damping. Making the choice of modal parameter estimation implies that the system is linear and time invariant. Consequently, the damping may not change with the excitation level $[12,22]$. Numerous parameter estimation techniques exist to extract the modal damping factor from an experimental modal analysis test. The most straightforward classification of these curve fitting methods is single-mode versus multiple-mode methods. Unlike multi-mode models, single-mode models show less accuracy but are also less time consuming due to their simplicity. Furthermore, singlemode models perform reasonably well for structures with lightly damped and well separated modes.

As it is the main purpose of this study to separate external damping losses from the material damping losses, a single-mode model is preferred over a multi-mode model for the simplicity of the data analysis throughout this paper. Moreover, conclusions related to external damping effects are generally applicable and do not depend on the modelling method. Among the single-mode models exist time- and frequency based methods for modal damping estimation. Both test methods are related to each other as both attribute 
all of the response to a single-mode in the vicinity of a resonance. The time-domain method is preferred over the frequency-domain method because the latter corresponds with very narrow resonant peaks at the response curve for lightly damped specimen, leading to insufficient accuracy [6,8]. The time-domain method is based on the exponential decay of the vibration when the specimen is left to vibrate freely after a resonance condition is induced. This type of decay is observed in a linear system which is described as a transfer function, and if only one single pair of complex poles dominates in the transfer function, which is true if the different modes are well separated, as described by Ewins [23]. The modal damping ratio is calculated from the logarithmic decrement, this is the natural logarithm of the amplitude ratio of $n$ successive cycles: $\delta=\frac{1}{n} \ln \left(\frac{A_{1}}{A_{n+1}}\right)$, with $A_{1}$ and $A_{n+1}$ the amplitude at the start respectively the end of these $n$ cycles in the decaying response. The decaying amplitude of the vibrating specimen, can be described as:

$$
x(t)=X \sin \left(\omega_{d} t\right) e^{-\omega_{n} \zeta t}
$$

With $\omega_{\mathrm{d}}$ the damped natural pulsation which equals the natural pulsation $\omega_{\mathrm{n}}$ for small damping ratio $\zeta=\frac{\delta}{2 \pi}, \zeta \ll 1$. The obtained modal dampingfactor is more accurate with increasing number of periods [17]. This statement is true in case of damping in a linear system, as stated in the beginning of this paragraph. In a nonlinear system, with amplitude dependent damping, the exponential decay should be subdivided into ranges which show approximate linear damping. The corresponding result is only the best value at some intermediate amplitude, e.g. geometric mean of extremes.

\subsection{Practical considerations}

The apparatus should be able to induce resonance conditions and to measure the specimen's free vibration when the excitation is dropped. Any test setup consists of (i) 
the specimen under test, (ii) the excitation source, (iii) the response measurement system, (iv) the specimen support system and (v) the assembly of all parts.

\subsubsection{Specimen excitation}

A popular method to excite the specimen is the impact hammer. Depending on the impact frequency spectrum, one or more resonant frequencies will be excited. As we aim to extract the damping properties via the logarithmic decrement of the decay plot, we wish to excite one mode at the time. The pulse force of the impact hammer is not able to excite higher resonant modes separately such that filtering and other signal analysis techniques would be required for our purpose. The latter however can introduce phase shifts and spectral leakage and as such distort the outcome.

The electromagnetic exciter or shaker has more operational flexibility but needs to be attached to the specimen. The problem of connecting the drive platform of the shaker to the specimen will be discussed in paragraph 2.2.3. Alternatively, a loudspeaker can be used to excite the specimen $[17,16,24]$. A low power loudspeaker as excitation source can be used; because damping is evaluated at resonance frequency and consequently little excitation energy is necessary to cause sufficiently large specimen response deformation. For systems with inherently high damping, or with an added damping treatment the loudspeaker may not produce strains of engineering interest. However, choosing a high power loudspeaker with a good audio sensitivity in the frequency range of interest will improve the measurement. This method also fulfils the requirement of the ASTM standard [25], that non-contact making devices are preferred to minimize external damping sources. Another advantage is that the excitation amplitude level can easily be modified. The exciters should yield an equal amplitude level if tests are repeated, to prevent an inconsistency in the results caused by nonlinear damping [11]. 
This is difficult to achieve with an impact hammer [15,26,8,7], unless the hammer is mounted to make a pendulum swing.

Taking into account all considerations, the loudspeaker excitation is preferred over other exciters and will be used as an excitation source for damping measurements within this paper.

\subsubsection{The response measurement system}

The choice of the transducers will be from the perspective of eliminating external damping sources. The ASTM standard [25] recommends non-contacting sensors. If any contact-making sensor is used it must be demonstrated whether the device, and especially the wiring, is a significant source of damping. Different measurement systems are used in literature: accelerometers [7,27,11,13], strain gauges [6,14], inductive distance probes [26], laser Doppler vibrometer [8,24,16,15,17], microscope [20], optical displacement followers [1,28] and microphones [29]. In paragraph 4, the effect on the damping measurement of three different sensors will be examined and guidelines will be formulated.

\subsubsection{Boundary conditions}

The specimen should be connected to the environment through a suspension system. This can be as a grounded or a free condition implementation. Vibration experiments are often performed on a cantilever beam configuration $[25,20,6,1,8,27,11,14]$. As described in the ASTM standard [25], the apparatus should consist of a rigid, massive test fixture which provides a clamp for the root end of the beam. However, a rigid cantilever beam fixture is difficult to realize. Any construction with bolted connections and specimen grip systems may have a significant contribution to damping $[23,14]$ because of the slipping at the grip system and vibrating bolts. Similar effects are 
observed when a cantilever beam is clamped onto the outgoing shaft of an electrodynamic shaker which is suited for low force purposes: the shaker armature is not a massive fixture and this consequently leads to erroneous results for the damping values and specimen resonant frequencies [30,31]. The problem will be more prominent if larger (heavier) specimens are tested because the clamping mechanism experiences larger reaction forces. Though, even having firm and heavy cantilever grips does not fully eliminate this effect, any grip with a sharp corner will produce a stress concentration which guarantees some micro-slipping. To eliminate unwanted damping due to an improper realization of the boundary conditions (cf. the cantilever setup) it is opted in this paper to suspend the sample under free boundary conditions, i.e. the specimen has no constrained degrees of freedom. A common method is the suspension of the sample with thin wires at the nodal points of the resonant mode shape of interest. Then, the wires will not interfere with the sample.

\subsection{Test specimens and sample preparation}

The specimens have square dimensions with different sizes which vary from $100 \times 100 \times 2 \mathrm{~mm}$ up to $350 \times 350 \times 2 \mathrm{~mm}$ in steps of $50 \mathrm{~mm}$. These specimens consist of cold rolled steel plate material made of construction steel S235. In addition, two types of steel alloys, annealed alloy steel 4130 and annealed stainless steel T-304 2B, with square dimensions $300 \times 300 \times 2 \mathrm{~mm}$ will be tested as well. Once the specimens are cut to the right dimensions, the only specimen preparation left is the attachment of the suspension wires with a small glue dot such that damage of the specimen is prevented. Nylon cords with a $0.3 \mathrm{~mm}$ diameter are used, which are sufficiently to carry the weight of the specimens. One could think of drilling small holes but these may change the dynamic properties of the specimen, especially when testing small and light weight specimens. 
Next, the response measurement sensor is mounted onto the sample and finally the specimen can be suspended in the vertical plane in front of the loudspeaker.

\begin{tabular}{l|ccc} 
& $\begin{array}{c}\text { Young's Modulus } \\
{[\mathrm{GPa}]}\end{array}$ & Poisson's ratio [-] & Mass density $\left[\mathrm{kg} / \mathrm{m}^{3}\right]$ \\
\hline S235 & $205-215$ & $0.285-0.295$ & $7800-7900$ \\
\hline alloy steel 4130 & $201-216$ & $0.285-0.295$ & $7800-7900$ \\
annealed & $190-203$ & & $7850-8060$ \\
stainless T-304 & & $0.265-0.275$ & \\
annealed 2B & &
\end{tabular}

\subsection{Test apparatus}

The specimen is excitated with a $60 \mathrm{~W}$ rms loudspeaker in the frequency range of 20$5500 \mathrm{~Hz}$ (type: SP-W65-SONO woofer). The excitation signal is generated by LabVIEW software and National Instruments (NI) analog output hardware (C-series module NI-USB 9263). The accelerometer is an IEPE (integrated electronic piezoelectric) sensor with a $100 \mathrm{mV} / \mathrm{g}$ sensitivity and $50 \mathrm{~g}$ limit, type PCB 352C65. Strain measurements are assessed with a Vishay strain gauge, type CEA-06-250UN350, which suits for steel specimens. For response measuring with the laser Doppler vibrometer (LDV), a Polytec OFV-5000 with the corresponding velocity decoder VD06 and OFV-534 sensor head is used. The full scale range is $\pm 500 \mathrm{~mm} / \mathrm{s}$ and the corresponding frequency range is $0-350 \mathrm{kHz}$. The output signal is within $\pm 10 \mathrm{~V}$. Data acquisition is done through NI C-series modules NI9215, NI9234 and NI9237 for the LDV device, the IEPE accelerometer and the strain gauge respectively.

Numerical analysis is done through Abaqus v6.11 Finite Element (FE) software for specimen mode shape visualization. The plate specimens are modelled with S8R thick 
shell elements and are given appropriate section properties (Young's modulus, Poisson's ratio and mass density).

\section{Test methodology}

The test methodology should meet the requirements of paragraph 2.1, which is about damping modelling in a single-mode approach.

In a first step, the test procedure seeks the first and second resonant frequencies of the specimen with a random noise audio signal excitation. The single-mode approach assumes that the modes of interest are well separated; two closely spaced frequencies will corrupt the observed response as the response due to the nearby mode moves in and out of phase with that of the intended mode [23]. This problem could arise because anisotropy is introduced in cold rolled specimens or if one side is slightly different in length from the other. This condition is approved through analysing the response frequency spectrum with FE software, no closely spaced frequencies are observed.

Then, the loudspeaker excites the specimen at the first or second resonant frequency with a harmonic sine wave. Once the specimen vibration is in a steady-state condition, the loudspeaker excitation is turned off and the attenuating vibration response of the specimen is measured. Care is taken to insure that pendulum oscillations do not corrupt the signal through high-pass filtering the signal at $5 \mathrm{~Hz}$. Each time five consecutive measurements are executed to calculate an average value and a standard deviation, such that the repeatability of the test method is determined.

Based on the mode shape of the resonant frequency of interest one can point the optimum location on the specimen for both the excitation source and response measurement. A mode shape consists of nodal and anti-nodal locations, i.e. points where the specimen does not deform respectively deforms maximally. It is advised to measure and excite at those anti-nodal points to obtain maximum sensitivity. The FE 
analysis reveals that the first mode shape for a square steel sample is a twisting mode, as visualised in Figure 1.a. Maximum deformation is found at the four corners of the plate, thus the loudspeaker's position and the vibration response location of the specimen will be at one of the four corners. Based on Figure 1.b, which depicts the second resonant mode shape, it follows that the loudspeaker's position and vibration response location is at the middle of one of the sides of the plate.

\section{Evaluation of the response measurement sensor on the specimen modal damping}

The aim of this analysis is to compare the measured vibration response of the specimen if three different sensors, both contact- and non-contact making sensors are used. Although it is generally known that contact making sensors should be avoided when testing low damped specimens, this analysis will quantify the sensor interference with the sample and its influence on the modal damping.

A square steel $\mathrm{S} 235$ sample with dimensions $300 \times 300 \times 2 \mathrm{~mm}$ is used. The specimen is suspended at the nodal points of the first mode shape, according to Figure 1.a. Two nylon cords with $400 \mathrm{~mm}$ length are attached at the sample, each at the middle of one side of the sample such that the sample hangs vertically. The sample is positioned at a distance of $80 \mathrm{~mm}$ from the loudspeaker. Measuring the vibration response at the specimen suspension points confirms that the nylon cords are attached at the nodal points because no response signal is measured at these locations.

\subsection{Accelerometer response measurement}

The most obvious method to measure structural vibrations is with an accelerometer because this sensor can easily be mounted onto the structure or specimen. A low accelerometer mass (compared to the mass of the structure under test) should be the main specification to avoid sensor interference with the dynamics of the specimen as the 
dynamic force $m \ddot{x}$ (with $\ddot{x}$ the acceleration and $m$ the sensor mass) is of importance when testing at (high) resonance frequencies. Dynamic interference from the accelerometer can be minimised if the sensor is placed near a nodal spot but this reduces the response sensitivity so it is opted to place the accelerometer at an anti-nodal point. Two sensor mounting configurations are assessed: the first one is to attach the sensor wire to the specimen with tape from the corner to the centre of the plate and the second method is to allow the wire to oscillate, thus not fixed to the vibrating specimen. The sensor itself is attached with wax onto the specimen.

Figure 2.a shows that once the specimen is at steady-state vibration, the loudspeaker excitation is switched off and subsequently the specimen vibrates in free condition. Figure 2.b depicts the power spectral density of the response signal in Figure 2.a. It illustrates that the response signal is purely harmonic with a resonant frequency of 78.7 $\mathrm{Hz}$, just as the excitation signal is harmonic.

In a first attempt to check for comparison between both test configurations, the vibration response of both configurations is plotted on top of each other. The envelope of the attenuating response signals is plotted in Figure 3 to facilitate the comparison. It is obvious that the sensor wire fixing method has a big influence on the response. Much more damping is present if the wire is attached to the specimen; cf. the solid line in Figure 3. The fixed wire will prevent specimen vibration and any rubbing from the wire on the specimen will add damping. This finding is confirmed if the damping ratio is numerically determined. The damping ratio $\zeta$, calculated between $8 \mathrm{~g}$ and $0.125 \mathrm{~g}$, is more than doubled if the sensor wire is attached to the specimen. The damping ratio $\zeta$ is $9.5 \cdot 10-4$ and $4.6 \cdot 10-4$ for the fixed wire respectively the loose wire. The damping value from the loose wire configuration will be the most correct one because this corresponds 
with the lowest damping value; higher damping values imply additional (unwanted) damping.

\subsection{Contactless response measurement}

The specimen's deflection can be measured with several noncontact transducers. The inductive or capacitive proximity probes show a high accuracy but rather obtain the measurement of a spot than the measurement of a point such that one cannot exactly distinguish at which location the response measurement takes place. This can be an issue if the nodal points of a resonant mode shape should be detected and if small specimen sizes have to be evaluated. Also, it should be considered that the magnetic field of an inductive proximity probe may disturb the damping measurement.

Another contactless measuring method is through the Doppler principle. Not the acceleration or the displacement, but the velocity is measured and a very small measurement region is assured because a laser beam points onto the specimen. Reflective tape is used to improve laser beam reflection. It is verified that this small piece of tape does not act as an added constrained-layer damper to the system. Tests with retro-reflective dust instead of tape give identical damping results. This test setup is shown in Figure 5. The specimen under test is placed between the loudspeaker and the laser beam generator from the LDV. The laser beam points at the tip of the sample; this assures maximum response sensitivity if the first mode shape is excited (as depicted in Figure 1.a). Also the loudspeaker is positioned near the corner of the plate for maximum excitation sensitivity.

The excitation is first increased until the tip response amplitude of $80 \mathrm{~mm} / \mathrm{s}$ is achieved and subsequently is switched off letting the specimen vibrate in free condition. The decaying response signal is initially analysed between the start and end amplitude of $80 \mathrm{~mm} / \mathrm{s}$ respectively $1.25 \mathrm{~mm} / \mathrm{s}$, giving a damping ratio value $\zeta$ of $9.45 \cdot 10-5$. If the 
corresponding exponential (Eq. (1)) is plotted onto the original response signal (see Figure 6), it is observed that the mathematical model does not fit exactly. This means that not one single exponential function fits the decaying amplitude. This indicates that non-linear damping is present: the damping ratio depends on the response amplitude. Because in paragraph 2.1 linear damping is assumed for damping modelling, it is necessary to define damping within specific limits where damping shows approximate linear behaviour. Theoretically, an infinite number of amplitude ranges is necessary to characterize the non-linear damping behaviour described by exponential decaying curves. Because of practical reasons, six amplitude ranges are found to be an acceptable compromise between modelling accuracy and analysis complexity. The overall response amplitude range from $80 \mathrm{~mm} / \mathrm{s}$ up to $1.25 \mathrm{~mm} / \mathrm{s}$ is divided into six equal amplitude intervals and for each segment an exponential is fitted following the relation in Eq. (1). Figure 7 plots a detailed view of two exponential curves in the range $80-40 \mathrm{~mm} / \mathrm{s}$ and $2.5-1.25 \mathrm{~mm} / \mathrm{s}$. It is clear that only one single exponential curve fits in the range of 80 $40 \mathrm{~mm} / \mathrm{s}$ (with the + marker type), the other curve (with the $\diamond$ marker type) which belongs to the other amplitude range clearly deviates.

The damping ratio varies from $13 \cdot 10-5$ to $8.04 \cdot 10-5$ in the amplitude range of $80-40$ $\mathrm{mm} / \mathrm{s}$ to $2.5-1.25 \mathrm{~mm} / \mathrm{s}$ respectively, larger response amplitudes correspond with larger damping values. Table 2 gives the average damping ratio as function of response amplitude and the corresponding standard deviation from five consecutive measurements. Within all response amplitude ranges diverges the theoretical exponential decay (Eq. (1)) maximally $2 \%$ from the measured response decay. This error is found to be acceptable related to other measurement errors described further in this document. Thus, it is decided not to have more response amplitude ranges. The very 
low standard deviation allows for accurate comparison among different damping values, if any differences are observed it will not be due to lack of measurement repeatability.

\begin{tabular}{c|c|c}
$\begin{array}{c}\text { Response amplitude } \\
{[\mathrm{mm} / \mathrm{s}]}\end{array}$ & $\begin{array}{c}\text { Average damping } \\
\text { ratio } \zeta\end{array}$ & Standard deviation \\
\hline $80 \rightarrow 40$ & $13 \cdot 10-5$ & $3.03 \cdot 10-7$ \\
$40 \rightarrow 20$ & $10.8 \cdot 10-5$ & $1.76 \cdot 10-7$ \\
$20 \rightarrow 10$ & $9.42 \cdot 10-5$ & $9.6 \cdot 10-7$ \\
$10 \rightarrow 5$ & $8.66 \cdot 10-5$ & $1.02 \cdot 10-7$ \\
$5 \rightarrow 2.5$ & $8.26 \cdot 10-5$ & $1.45 \cdot 10-7$ \\
$2.5 \rightarrow 1.25$ & $8.04 \cdot 10-5$ & $4.89 \cdot 10-7$
\end{tabular}

Table 2: Nonlinear damping ratio: damping value depends on response amplitude

\subsection{Strain gauge response measurement}

Another response measuring method is strain measurement. The specimen deformation, cf. strain, can be measured by means of a strain gauge mounted at a high strain region of the specific resonant mode shape. This method should be beneficial in eliminating dynamic interference with the sample because the mass of the strain gauge is much lower compared to the accelerometer mass, the sensor versus plate mass ratio for the strain gauge respectively the accelerometer is $0.7 \cdot 10-4$ and $14 \cdot 10-4$. The strain gauges are attached to the specimen with the strain gauge adhesive M-Bond 200 from Vishay. The effect of a strain gauge is examined on the first mode shape of the plate. Both the shear- and longitudinal strain is measured. Additionally with the strain gauge sensor is the response also measured with the Doppler vibrometer, in this way it is possible to correlate the strain response with the velocity response which makes comparison with the other response measuring methods possible.

\subsubsection{Shear strain response}

Figure 4.a and Figure 4.b show respectively the shear- and longitudinal strain level for the first mode shape of the square plate. The shear strain component E12 dominates the 
longitudinal strain level E11, and the maximum shear strain is situated at the centre, at the nodal region. The strain gauge is positioned at an angle of 45 degrees on the plate and is configured in a quarter bridge configuration. Although a half- or full Wheatstone bridge configuration shows benefits in signal output level and noise reduction, a quarter bridge requires less wiring to connect the strain gauge and consequently will be beneficial in reducing external damping sources. A half bridge configuration would require two strain gauges positioned in the centre at each plate side and is a possible source of added damping due to wire vibration.. Anyway, the loading effect, which is significant with accelerometer testing, will be minimised as the strain gauge is mounted close to a nodal point. As with the accelerometer configuration, two wire-fixing configurations are tried out, a fixed and loose wire mounting.

From the method with the strain gauge lead wires fixed to plate show Figure 8.a and Figure $8 . b$ the velocity response respectively the strain response. The original envelope strain response signal, marked in grey colour in Figure 8.b, shows a lot of noise because the strain level is relatively low. Therefore, it is chosen to smooth the envelope curve with a running average of 50 points, the results is the thick black curve on top of the grey curve in Figure 8.b. The excitation has increased over $80 \mathrm{~mm} / \mathrm{s}$ velocity response and then is switched off. The corresponding shear strain at $80 \mathrm{~mm} / \mathrm{s}$ is $10.4 \mu$ strain. In analogy with previous analysis is the velocity response signal subdivided into amplitude intervals. The measured damping ratio is found in column B of.Table 3. Similar results are obtained if the strain gauge lead wires are not fixed to the plate. The decaying signal from the strain gauge and the Doppler vibrometer are given respectively in Figure 9.a and Figure 9.b. The corresponding damping ratios are given in column $\mathrm{C}$ of Table 3. 


\subsubsection{Longitudinal strain response}

Figure 4.b depicts near the sides of the plate spots of longitudinal E11 strain. The level is significantly lower than the shear strain component, but the main difference with the shear strain response at the centre of the plate is that these spots are located at anti-nodal points. This could introduce additional lead wire vibration, and consequently increased system damping. One strain gauge is mounted at both sides of the plate (making two in total, each measuring $+\varepsilon$ and $-\varepsilon$ ), and are placed in a half Wheatstone bridge configuration. A half bridge is preferred over a quarter bridge to amplify the output signal when low strain levels are expected. Furthermore, the sensor lead wire is not fixed to the plate with glue dots. Figure 10.a and Figure 10.b depict the attenuating velocity response, respectively the strain response. The noise level on this strain signal is much lower compared to the shear strain signal (Figure 9.a and Figure 9.b); this is due to the half Wheatstone bridge configuration. Column D in Table 3 shows the damping ratio values.

\subsection{Summary}

Table 3Error! Reference source not found. gives an overview of all the response measuring methods assessed in Paragraph 4.1, 4.2 and 4.3. These tests were performed on the S235 sample with dimensions $300 \times 300 \times 2 \mathrm{~mm}$ which is supported with $400 \mathrm{~mm}$ suspension cords at the nodal point of the first resonant mode shape. The acceleration response is transformed to velocity response, making a comparison with the LDV method possible. Since the response signal is harmonic, acceleration $\ddot{x}$ and velocity $\dot{x}$ relate as $\dot{\mathrm{x}}=\ddot{\mathrm{x}} / 2 \pi \mathrm{f}$, with $f$ the resonant frequency.

It is clear that the accelerometer based response shows the largest contribution in added damping. A fixed or loose lead wire configuration for the acceleration sensor does not give the same correctness as is obtained with the strain gauge configurations. The added 
damping source when using accelerometers is due to the stiffer coax wire showing higher resistance against vibration and the higher sensor mass. The closest approximation to the reference value from the LDV is obtained with placing the strain gauge close to a nodal point and a loose wire configuration. If the strain gauge is positioned at an anti-nodal point, still is the obtained damping value closer to the reference value than the accelerometer based damping value. Based on these results it can be concluded that non-contact making transducers (here: LDV) suit for this type of damping measurement because these do not interfere with the sample and consequently do not contaminate the overall damping value. Other types of optical sensors probably will be appropriate too. However, in many applications it is not possible to have a clear normal LDV path to the observation point. If so, it is preferred to use the strain gauges with loose wires near a nodal region over the accelerometer. If no other sensor than the accelerometer is possible, it should be placed near a nodal region but one should take into account the reduced output voltage of the accelerometer.

\begin{tabular}{c|c|c|c|c|c|c}
$\begin{array}{c}\text { Velocity response } \\
\text { amplitude }[\mathrm{mm} / \mathrm{s}]\end{array}$ & $\mathrm{A}$ & $\mathrm{B}$ & $\mathrm{C}$ & $\mathrm{D}$ & $\mathrm{E}$ & $\mathrm{F}$ \\
\hline $80 \rightarrow 40$ & 13.2 & 14.7 & 13.7 & 18.8 & 47.5 & 35.5 \\
$40 \rightarrow 20$ & 10.9 & 12.1 & 11.1 & 17.2 & 82.4 & 44.5 \\
$20 \rightarrow 10$ & 9.49 & 10.6 & 9.55 & 16.7 & 106 & 51.8 \\
$10 \rightarrow 5$ & 8.71 & 9.76 & 8.70 & 16.7 & 102 & 57.4 \\
$5 \rightarrow 2.5$ & 8.35 & 9.36 & 8.26 & 16.9 & 107 & 67.4 \\
$2.5 \rightarrow 1.25$ & 8.1 & 9.16 & 8.08 & 17.0 & 125 & 78.3
\end{tabular}

Table 3: Overview of damping values $\zeta\left(\cdot 10^{-5}\right)$ with different response measuring methods for the first resonance mode. A: Doppler vibrometer; B: shear strain with fixed wire; C: shear strain with loose wire; D: longitudinal strain with loos wire; E: accelerometer with fixed wire; F: accelerometer with loose wire 


\section{Sensitivity analysis on external factors affecting the damping test setup}

Three different external damping contributions will be examined into detail within this paragraph. Construction steel S235 samples will be used and only the specimen dimensions will be changed, the square geometry remains.

\subsection{Air damping}

It is not straightforward to determine effective numerical values for the air damping contribution to the overall damping value. Air damping estimations from literature focus on comparing damping tests assessed in a vacuum (or lowered air pressure) environment with results from an ambient air pressure environment found experimentally or through the calculation of the fluid-structure interaction by means of Reynolds numbers, [20,26,1,17,7,32]. The analysis here differs because several damping test configurations in ambient air pressure conditions are compared to each other as air damping is always present in real life applications. The distance between the test specimen and the loudspeaker is varied to influence the specimen's surrounding air. If the distance increases, the air vibrates in a 'free environment'. If the distance decreases, the air constantly moves in the space between the loudspeaker and the specimen; i.e. a cushioning effect of the air is created.

Three different sample sizes are tested on their air damping contribution, namely 100x100x2 mm, 200x200x2 mm and 300x300x2 mm. The specimens are suspended at the nodal points of the first mode shape with nylon wires of $400 \mathrm{~mm}$ length. Five different loudspeaker-specimen distances are selected, and each time the damping is measured. Table 4 reports the absolute damping values, each obtained from tests at a different distance between the loudspeaker and the sample. Additionally, the results are subdivided into different response amplitude ranges because of the non-linear damping. 
Firstly, from Table 4 it is observed that the measured damping ratio decreases to an asymptotic value (cf. the damping value which belongs to the $300 \mathrm{~mm}$ distance) with increasing space between the loudspeaker and the specimen. Secondly, the ratio of the separation distance between loudspeaker and specimen is found to be the driven parameter for the air damping interference. Table 5 reports the relative difference between the reference damping value at $300 \mathrm{~mm}$ and the damping value obtained at another sample-loudspeaker distance. The percentage air damping contribution is approximately independent of the response amplitude and increases linear with the ratio of separation distance.

The third observation reveals that the relative air damping contribution at different separation distance ratios is approximately independent of the sample size, this is given in Table 6 . The value between round brackets gives the real distance ratio, the reference value is smaller $(80 \mathrm{~mm}$ and $150 \mathrm{~mm}$ instead of $300 \mathrm{~mm}$ ) for the smaller samples due to lack of excitation power at larger distances. The relative air damping contributions in Table 6 are representative for the whole response amplitude range; in other words, the air damping contribution hardly changes with the response level.

For the following experiments is a ratio of separation distance of 3.75 chosen such that the relative contribution remains below 5\% (see Table 5 and Table 6). This value is a compromise between air damping interference and the excitation level. A larger distance implies less air contamination but also less excitation power that reaches the sample. Probably, even if the surrounding air does not interfere with other objects (the loudspeaker), there still will be air damping contribution. This aspect has been investigated by Baker et. al. [10], the auhors have drawn conclusions about air damping contribution from tests at aluminum beam specimens in a cantilever configuration. The 
trend is (i) that for large bending amplitudes air damping is increasing in proportion to the amplitude and (ii) that for small amplitudes the air damping loss factor appears to approach a limit which is independent of amplitude. It is also noted that the mode shape is of relatively little effect on the air damping ratio. Because these results are based on tests on cantilever beam samples it might be possible that these conclusions are not fully valid for plate specimens. The contribution of air damping in atmospheric pressure for plate specimens has been investigated by De Visscher et. al. [17]. A semi-empirical formula estimates the air damping ratio, the latter is inversely proportional with the specific weight $\rho\left[\mathrm{kg} / \mathrm{m}^{3}\right]$, the thickness $t[\mathrm{~m}]$ and the square root of the resonance frequency fres $[\mathrm{Hz}]$ of the plate. This formula might be applicable for the test results in this paper because De Visscher et. al. [17] make use of the test same methodology for damping measurements of plates. As no dependence on vibration amplitude is mentioned, there might be a correlation with results from Baker et. al. [10] which state that for low vibration amplitudes the air damping contribution is constant.

The conclusion here is that one should take into consideration that any object close to the specimen under test may impair the damping results. Therefore, it is necessary to do this sensitivity analysis to prevent incorrect results.

Damping ratio $\zeta(\cdot 10-5)$

\begin{tabular}{c|ccccc}
\hline \multirow{2}{*}{$\begin{array}{c}\text { Response } \\
\text { ampltidue }[\mathrm{mm} / \mathrm{s}]\end{array}$} & \multicolumn{4}{|c}{ Distance between sample and loudspeaker } \\
\cline { 2 - 6 } & $20 \mathrm{~mm}$ & $40 \mathrm{~mm}$ & $80 \mathrm{~mm}$ & $150 \mathrm{~mm}$ & $300 \mathrm{~mm}$ \\
\hline
\end{tabular}




\begin{tabular}{c|ccccc}
$2.5 \rightarrow 1.25$ & 10.2 & 8.77 & 8.10 & 7.95 & 7.89 \\
$5 \rightarrow 2.5$ & 10.4 & 8.93 & 8.35 & 8.15 & 8.05 \\
$10 \rightarrow 5$ & 10.8 & 9.38 & 8.71 & 8.51 & 8.33 \\
$20 \rightarrow 10$ & 11.5 & 10.1 & 9.49 & 9.23 & $/$ \\
$40 \rightarrow 20$ & 12.9 & 11.6 & 10.9 & 10.4 & $/$ \\
$80 \rightarrow 40$ & 15.3 & 14.1 & 13.2 & $/$ & $/$
\end{tabular}

Table 4: Damping values at different sample-loudspeaker distances for the largest sample 300x300x2 mm

\begin{tabular}{|c|c|c|c|c|}
\hline \multirow{2}{*}{$\begin{array}{c}\text { Response } \\
\text { amplitude }[\mathrm{mm} / \mathrm{s}]\end{array}$} & \multicolumn{4}{|c|}{ Ratio of seperation distance } \\
\hline & $\begin{array}{c}2 \\
(300 / 150)\end{array}$ & $\begin{array}{c}3.75 \\
(300 / 80)\end{array}$ & $\begin{array}{c}7.5 \\
(300 / 40)\end{array}$ & $\begin{array}{c}15 \\
(300 / 20)\end{array}$ \\
\hline $2.5 \rightarrow 1.25$ & $-1 \%$ & $-3 \%$ & $-11 \%$ & $-29 \%$ \\
\hline $5 \rightarrow 2.5$ & $-1 \%$ & $-4 \%$ & $-11 \%$ & $-29 \%$ \\
\hline $10 \rightarrow 5$ & $-2 \%$ & $-5 \%$ & $-13 \%$ & $-29 \%$ \\
\hline
\end{tabular}

Table 5: Relative air damping contribution for the largest sample 300x300x2 mm

\begin{tabular}{c|cccc}
\multirow{2}{*}{ Sample size $[\mathrm{mm}]$} & \multicolumn{4}{|c}{ Ratio of seperation distance } \\
\cline { 2 - 5 } & 2 & 3.75 & 7.5 & 15 \\
\hline \multirow{2}{*}{$100 \times 100 \times 2$} & $\mathbf{- 1 \%}$ & $\mathbf{- 3 \%}$ & $\mathbf{- 8 \%}$ & $/$ \\
& $(80 / 40)$ & $(80 / 20)$ & $(80 / 10)$ & \\
$200 \times 200 \times 2$ & $\mathbf{- 1 \%}$ & $\mathbf{- 3 \%}$ & $\mathbf{- 1 2 \%}$ & $/$ \\
$300 \times 300 \times 2$ & $(150 / 80)$ & $(15040)$ & $(150 / 20)$ & $\mathbf{- 2 9 \%}$ \\
& $\mathbf{- 1 \%}$ & $-3 \%$ & $\mathbf{- 1 1 \%}$ & $(300 / 20)$
\end{tabular}

Table 6: Relative air damping contribution for amplitude range $2.5-1.25 \mathrm{~mm} / \mathrm{s}$

\subsection{Suspension: effect of length and nodal-or anti-nodal position}

In paragraph 2.2.3 it was advised to attach nylon wires at the nodal points of the particular mode shape to suspend the specimen. The influence of the suspension length within this situation is determined by measuring the specimen damping with three different suspension lengths: $400 \mathrm{~mm}, 200 \mathrm{~mm}$ and $100 \mathrm{~mm}$. The relative error on the damping ratio between two suspension lengths is used as follows: $200 \mathrm{~mm}$ vs. $400 \mathrm{~mm}$, $100 \mathrm{~mm}$ vs. $400 \mathrm{~mm}$ and $100 \mathrm{~mm}$ vs. $200 \mathrm{~mm}$. The same three specimen sizes from the air damping experiment are used. The damping values for all combinations of specimen size and specimen length, for both the first and second mode shape are given in Table 7 and Table 8 respectively. For the three sample sizes, there is hardly any difference (for 
both the first and second mode shape), the relative error is about 1\%. Except for the first mode shape of the smallest sample $(100 \times 100 \times 2 \mathrm{~mm})$ which shows a $4 \%$ higher damping value for the $100 \mathrm{~mm}$ suspension length compared to the $400 \mathrm{~mm}$ suspension length. This could be due to small misalignment of the nylon cords onto the specimen, but such a small error is still acceptable. The smallest damping value, corresponding with the $400 \mathrm{~mm}$ suspension length, is believed to be the right one because a higher value means that additional damping effects take part.

Although it is generally understood that in free vibration experiments the specimen should be suspended at its nodal points, still some work from literature can be found which does not take this into consideration [33,15]. Possible reasons are: (i) the specimen can only be suspended in one manner due to other constraints or (ii) damage to the specimen must be avoided because of suspension mounting.

From this point of view, the goal of this sensitivity analysis is to assess the quantitative error on the overall damping value if the suspension cords are attached at anti-nodal positions. Two different types of dissipation effect can be distinguished in specimen suspension. Suspension threads can add damping by local frictional effects if there is any contact, and they also add damping by transporting energy away by wave propagation along the threads. The first is governed by details around the glue joint, the second by the wave impedance of the threads.

As with previous experiments, three different sample sizes are examined for their sensitivity to anti-nodal suspension. Furthermore, three different suspension lengths (100 mm, $200 \mathrm{~mm}$ and $400 \mathrm{~mm}$ ) are taken into consideration as it is believed this may have an effect also.

Damping from anti-nodal suspension gives additional damping compared with nodal suspension, . Table 7 and Table 9 should be compared for differences between nodal 
and anti-nodal suspenstion at the first mode shape, Table 8 and Table 10 depict the damping values for the second mode shape. From almost all test cases it is observed that if the response tip velocity increases, the relative difference between damping from nodal and anti-nodal position reduces. This outcome is most pronounced for the smallest suspension length of $100 \mathrm{~mm}$. A possible explanation is that a small response amplitude corresponds with a lower damping ratio (Table 2), and thus the added damping due to the suspension has a larger effect in terms of percentage. When looking more into detail, it can be seen that the smallest sample $(100 \times 100 \times 2 \mathrm{~mm})$ is most susceptible to anti-nodal suspension location and this for all suspension lengths (large \% errors). For the smallest specimen size combined with a $100 \mathrm{~mm}$ suspension length the relative error is in the range of $62 \%$ to $40 \%$, the $400 \mathrm{~mm}$ suspension gives errors up to $90 \%$. Thus, increasing the suspension length can have a positive or a negative effect depending on the dimensions of the specimens.

Similar reasoning is valid for damping measurements at the second resonant mode shape.

\begin{tabular}{|c|c|c|c|c|c|c|c|c|c|}
\hline $\begin{array}{l}\text { suspension length } \\
{[\mathrm{mm}]}\end{array}$ & \multicolumn{3}{|c|}{100} & \multicolumn{3}{|c|}{200} & \multicolumn{3}{|c|}{400} \\
\hline specimen size $[\mathrm{mm}]$ & 100 & 200 & 300 & 100 & 200 & 300 & 100 & 200 & 300 \\
\hline Frequency $[\mathrm{Hz}]$ & 680.4 & 170.9 & 78.9 & 680.5 & 170.9 & 78.8 & 680.5 & 170.9 & 78.8 \\
\hline $\begin{array}{c}\text { Response amplitude } \\
{[\mathrm{mm} / \mathrm{s}]}\end{array}$ & & & & & & & & & \\
\hline
\end{tabular}




\begin{tabular}{l|ccc|ccc|ccc}
$2.5 \rightarrow 1.25$ & 6.75 & $/$ & 8.13 & 6.66 & 7.64 & 8.09 & 6.49 & 7.61 & 8.04 \\
$5 \rightarrow 2.5$ & 6.95 & 7.91 & 8.33 & 6.89 & 7.79 & 8.28 & 6.73 & 7.73 & 8.26 \\
$10 \rightarrow 5$ & 7.28 & 8.19 & 8.71 & 7.14 & 8.11 & 8.71 & 7.04 & 8.05 & 8.66 \\
$20 \rightarrow 10$ & 7.96 & 8.75 & 9.48 & 7.91 & 8.71 & 9.47 & 7.65 & 8.64 & 9.42 \\
$40 \rightarrow 20$ & 9.19 & 9.72 & 10.9 & 9.10 & 9.77 & 10.8 & 8.78 & 9.71 & 10.8 \\
$80 \rightarrow 40$ & 11.2 & 11.4 & 13.2 & 11.2 & 11.3 & 13.1 & $/$ & 11.4 & 13.0
\end{tabular}

Table 7: Damping values from specimen suspension at nodal position of first mode shape for different suspension lengths and specimen size

\begin{tabular}{c|ccc|ccc|ccc}
$\begin{array}{c}\text { suspension length } \\
{[\mathrm{mm}]}\end{array}$ & \multicolumn{3}{|c|}{100} & & \multicolumn{3}{|c|}{200} & & \multicolumn{3}{|c}{400} \\
\hline specimen size [mm] & 100 & 200 & 300 & 100 & 200 & 300 & 100 & 200 & 300 \\
\hline Frequency [Hz] & 949.0 & 239.0 & 110.4 & 949.1 & 239.0 & 110.4 & 949.1 & 239.0 & 110.4 \\
\hline Response amplitude & & & & & & & & & \\
{$[\mathrm{mm} / \mathrm{s}]$} & & & & & & & & & \\
$2.5 \rightarrow 1.25$ & 8.20 & 9.64 & 11.1 & 8.17 & 9.47 & 11.0 & 8.21 & 9.43 & 10.9 \\
$5 \rightarrow 2.5$ & 8.40 & 9.82 & 11.4 & 8.41 & 9.73 & 11.3 & 8.36 & 9.70 & 11.2 \\
$10 \rightarrow 5$ & 8.83 & 10.3 & 11.9 & 8.79 & 10.2 & 11.7 & 8.79 & 10.1 & 11.7 \\
$20 \rightarrow 10$ & 9.60 & 11.1 & 12.9 & 9.57 & 11.2 & 12.7 & 9.59 & 11.1 & 12.7 \\
$40 \rightarrow 20$ & 11.1 & 12.6 & 14.7 & 11.0 & 12.8 & 14.4 & 11.1 & 12.7 & 14.5 \\
$80 \rightarrow 40$ & 13.5 & 15.3 & 17.7 & 13.4 & 15.3 & 17.1 & 13.5 & 15.2 & 17.5
\end{tabular}

Table 8: Damping values for specimen suspension at nodal position of second mode shape for different suspension lengths and specimen size

\begin{tabular}{|c|c|c|c|c|c|c|c|c|c|}
\hline $\begin{array}{c}\text { suspension length } \\
{[\mathrm{mm}]}\end{array}$ & \multicolumn{3}{|c|}{100} & \multicolumn{3}{|c|}{200} & \multicolumn{3}{|c|}{400} \\
\hline specimen size $[\mathrm{mm}]$ & 100 & 200 & 300 & 100 & 200 & 300 & 100 & 200 & 300 \\
\hline Frequency [Hz] & 680.3 & 171 & 78.9 & 680.2 & 170.9 & 78.8 & 680.4 & 170.9 & 78.8 \\
\hline $\begin{array}{c}\text { Response amplitude } \\
{[\mathrm{mm} / \mathrm{s}]}\end{array}$ & & & & & & & & & \\
\hline
\end{tabular}




\begin{tabular}{l|lll|lll|lll}
$2.5 \rightarrow 1.25$ & 10.9 & 8.23 & 9.89 & 9.57 & 7.59 & 8.37 & 12.3 & 7.60 & 8.38 \\
$5 \rightarrow 2.5$ & 11.1 & 8.44 & 10.2 & 9.73 & 7.83 & 8.56 & 12.4 & 7.77 & 8.60 \\
$10 \rightarrow 5$ & 11.5 & 8.73 & 10.4 & 10.0 & 8.19 & 8.95 & 12.7 & 8.08 & 8.99 \\
$20 \rightarrow 10$ & 12.1 & 9.12 & 11.2 & 10.6 & 8.87 & 9.69 & 13.4 & 8.66 & 9.76 \\
$40 \rightarrow 20$ & 13.4 & 10.1 & 12.6 & 11.8 & 9.82 & 11.0 & 14.6 & 9.71 & 11.1 \\
$80 \rightarrow 40$ & 15.7 & 11.7 & 14.7 & 14.1 & 11.4 & 13.2 & 16.5 & 11.5 & 13.5
\end{tabular}

Table 9: Damping values from specimen suspension at anti-nodal position of first mode shape for different suspension lengths and specimen size

\begin{tabular}{|c|c|c|c|c|c|c|c|c|c|}
\hline $\begin{array}{l}\text { suspension length } \\
{[\mathrm{mm}]}\end{array}$ & \multicolumn{3}{|c|}{100} & \multicolumn{3}{|c|}{200} & \multicolumn{3}{|c|}{400} \\
\hline specimen size $[\mathrm{mm}]$ & 100 & 200 & 300 & 100 & 200 & 300 & 100 & 200 & 300 \\
\hline Frequency $[\mathrm{Hz}]$ & 948.9 & 239.1 & 110.5 & 949 & 239.1 & 110.5 & 949.1 & 239.1 & 110.5 \\
\hline $\begin{array}{c}\text { Response amplitude } \\
{[\mathrm{mm} / \mathrm{s}]}\end{array}$ & & & & & & & & & \\
\hline $2.5 \rightarrow 1.25$ & 14.9 & 9.72 & 11.9 & 10.9 & 9.68 & 11.2 & 11.0 & 9.63 & 11.0 \\
\hline $5 \rightarrow 2.5$ & 15.1 & 9.90 & 12.2 & 11.0 & 9.89 & 11.4 & 11.1 & 9.90 & 11.3 \\
\hline $10 \rightarrow 5$ & 15.5 & 10.4 & 12.7 & 11.4 & 10.4 & 11.9 & 11.6 & 10.4 & 11.8 \\
\hline $20 \rightarrow 10$ & 16.3 & 11.3 & 13.7 & 12.1 & 11.4 & 13.0 & 12.4 & 11.4 & 12.8 \\
\hline $40 \rightarrow 20$ & 17.7 & 13.0 & 15.4 & 13.3 & 13.1 & 14.8 & 13.9 & 12.8 & 14.6 \\
\hline $80 \rightarrow 40$ & 20.1 & 15.5 & 18.3 & / & 15.8 & 17.9 & 16.5 & 15.4 & 17.5 \\
\hline
\end{tabular}

Table 10: Damping values for specimen suspension at anti-nodal position of second mode shape for different suspension lengths and specimen size

\subsection{Summary}

From the previous sensitivity analysis on unwanted damping effects it can be concluded that air damping at atmospheric conditions may have a significant influence on the system damping. This effect can easily be minimized by removing other (measurement) devices away from vibrating specimen. Another finding is the independence of the damping on the suspension length if the specimen is suspended at the nodal points of the resonant mode shape. Other locations for the suspension cords can yield distorted 
damping values. In general it can be concluded that anti-nodal suspension should be avoided when testing small specimen sizes, larger specimens experience less effect.

\section{Influence of specimen material -and geometry on damping}

In the introduction of the paper is stated that the outcome of the damping measurement depends on the initial stress-strain state of the structure. This is only valid if all other damping sources are eliminated, and consequently the outcome is related to material damping only. The test configuration described in this paper reduces added damping sources due to (i) the response sensor choice and sensor attachment, (ii) the specimen suspension, (iii) the position of the test sample near other (measurement) devices to eliminate air pumping and (iv) it is found that air damping adds a constant value to the measured damping ratio for this test configuration. This leads to an optimized test setup in reducing external damping sources. Therefore several experiments are performed to illustrate the efficiency of the test method. One experiment evaluates damping through changing the dimensions of samples of the same material. Another experiment evaluates damping with samples of equal dimensions but manufactured from three different steel alloys.

All samples are suspended at the nodal points with $400 \mathrm{~mm}$ nylon suspension cords.

\subsection{Damping as function of sample size}

Changing the dimensions of the sample does not change the inherent material damping. Though, it alters the initial stress-strain state of the sample and therefore will different sample sizes show other damping characteristics.

S235 steel samples from $100 \times 100 \times 2 \mathrm{~mm}$ to $350 \times 350 \times 2 \mathrm{~mm}$ with increasing steps of $50 \mathrm{~mm}$ are used for this analysis. The damping ratio is plotted as function of sample size area, given that the sample shape and thickness remains. Plotting the damping ratio as 
function of frequency makes no sense because changing the dimension changes both the frequency and the stress-strain state in the material.

At first is the damping ratio for the first mode shape determined. As can be seen in Figure 11 the damping ratio first shows a linear increase with sample area, and further shows a steeper increase. This trend is observed for all velocity amplitude ranges. It is noted that this observation cannot be extrapolated to other materials. Each material has its own damping law and therefore will show other damping characteristics as function of frequency and sample size.

Another visualization of the damping ratio for the first respectively the second mode shape is shown in Figure 12.a and Figure 12.b for three different sample sizes. The abscissa data points in both figures correspond with the highest value of each amplitude range (e.g. $40 \mathrm{~mm} / \mathrm{s}$ is the damping value for the $40-20 \mathrm{~mm} / \mathrm{s}$ response amplitude

range), cf. Table 2. It is clear that the second mode shape has much larger damping values than the first mode shape, but the trend as function of the response amplitude is the same for both. The damping increases with the response amplitude; a trend which is valid for all three sample sizes. Due to lack of sound intensity from the loudspeaker it is impossible to achieve higher tip velocities but it is expected that damping keeps increasing with larger amplitude level.

\subsection{Damping for different steel alloys}

In contrast with testing the same material at different sizes investigates this analysis the effect of different steel alloys with the same sample dimensions. This will reveal information about the inherent material damping because the initial stress-strain state is equal for the samples. In addition to the S235 steel, two steel alloy samples with dimension 300x300x2 mm will be tested; their specifications are given in Table 1. Table 11 gives an overview of the damping ratio for the three different steel alloys for the first 
and second mode shape as function of the response velocity amplitude. In contrast with all previous results in this work, it is observed that linear damping is present, for both steel alloy samples and for both the first and second resonant mode shape. The annealed 304-2B sample has an average damping ratio of $4.86 \cdot 10^{-5}$ and $5.3 \cdot 10^{-5}$ for the first, respectively the second mode shape. The damping ratio for the AISI 4130 sample is more than doubled, $10.2 \cdot 10^{-5}$ for the first mode shape and $13.4 \cdot 10^{-5}$ for the second mode shape. The resonance frequencies of the S235 and AISI 4130 sample are approximately the same and also their damping values are within the same range. The annealed 304-2B alloy has lower resonance frequencies, and the damping values are remarkably lower compared to the other. Although all samples are predominantly ferrous alloys, they definitely show different damping characteristics: the damping value can be significantly larger or smaller and linear -or nonlinear damping is possible. It is clear that damping values reported in literature should be applied in the same conditions as it was measured and not generalised to other applications.

\begin{tabular}{c|cc|cc|cc} 
& \multicolumn{5}{|c}{ Damping ratio $\zeta(\cdot 10-5)$} \\
\hline Specimen material & \multicolumn{2}{|c|}{ S235 } & \multicolumn{2}{|c}{ AISI 4130 } & Annealed 304-2b \\
\hline $\begin{array}{c}\text { First and second } \\
\text { resonant frequency [Hz] }\end{array}$ & 78.7 & 110.5 & 72.2 & 112.3 & 66.6 & 99.5 \\
\hline Response amplitude & & & & & & \\
{$[\mathrm{mm} / \mathrm{s}]$} & & & & & & \\
$2.5 \rightarrow 1.25$ & 8.04 & 10.9 & 10.1 & 13.4 & 4.86 & 5.22 \\
$5 \rightarrow 2.5$ & 8.26 & 11.2 & 10.1 & 13.3 & 4.86 & 5.30 \\
$10 \rightarrow 5$ & 8.66 & 11.7 & 10.1 & 13.3 & 4.84 & 5.28 \\
$20 \rightarrow 10$ & 9.42 & 12.7 & 10.2 & 13.4 & 4.85 & 5.25 \\
$40 \rightarrow 20$ & 10.8 & 14.5 & 10.4 & 13.6 & 4.87 & \\
$80 \rightarrow 40$ & 13.0 & 17.5 & 10.7 & 14.1 & 4.94 &
\end{tabular}


Table 11: Comparison of damping ratio for different steel (alloy) materials

\section{Conclusion}

This work has pointed several possible causes of errors which can slip into damping measurements if material damping from lightly damped specimens is determined through the free vibration time-domain method. The influence of some common parameters is quantified in terms of percentages, allowing the user to accept or reject the contaminating influence of the parameter on the overall measured damping value. From wired and wireless response measurement sensors it is observed that definitely contactless sensors must be used, if not, it is advised (i) to position the response measuring sensor near an anti-nodal spot, (ii) to use flexible sensor wiring and (iii) the mass of the response sensor should be very low compared to the specimen mass. A similar argument holds for the specimen excitation method, i.e. contactless excitation is preferred (e.g. through a loudspeaker) and when not feasible, one should examine the effect the clamping mechanism of the specimen onto the excitation device on the measured damping value. Also the air damping effect at atmospheric conditions is considered and can partly be eliminated if no (measurement) devices are placed nearby the sample under test. Any element close to the specimen may disturb the damping measurement; errors up to $16 \%$ are possible. Furthermore, the best way to suspend the specimen is in a free vibration condition by attaching thin wires at the nodal points of the resonant mode shape of interest. If not, the measured damping values may be totally wrong. Especially small size samples are very susceptible to this; errors up to $82 \%$ are found with this experimental setup. Based on these results an appropriate damping test setup is configured which minimizes the influence of external damping losses.

This work has also investigated the influence of specimen material -and geometry on the damping value. From tests on square steel samples made of S235 construction steel 
it can be concluded that the total system damping capacity significantly depends on the excitation level and the specimen size: high excitation amplitudes correspond with high damping values and larger specimens cause larger damping values. But, these findings are not confirmed by tests on other materials. Steel alloy samples (AISI4130 and annealed 304-2B) show other damping characteristics than the S235 samples. It can be concluded that material damping is case/specimen specific and the use of damping results from literature should be done with the utmost precaution.

\section{References}

[1] N. Granick and J. E. Stern, "Material damping of aluminum by a resonant- dwell technique," National Aeronautics and Space Administration, NASA Technical Note 1965.

[2] J. Zhang, R. J. Perez, and Lavernia E. J., "Documentation of damping capacity of metallic, ceramic and metal-matrix composite materials," Journal of Materials Science, vol. 28, pp. 2395-2404, 1993.

[3] M Colakoglu and KL Jerina, "Material damping in 6061-T6511 aluminium to assess fatigue damage," Fatigue \& Fracture of Engineering Materials I\& Structures, vol. 26, no. 1, pp. 9-84, January 2003.

[4] Clarence W. de Silva, Vibration and Shock Handbook, Clarence W. de Silva, Ed.: CRC Press - Taylor $\backslash \&$ Francis Group, 2005.

[5] R.D. Adams and D.G.C. Bacon, "Measurement of the flexural damping capacity and dynamic Young's modulus of metals and reinforced plastics," Journal of Physics D: Applied Physics, vol. 6, no. 1, p. 27, 1973.

[6] R. Gibson and R. Plunkett, "A forced-vibration technique for measurement of 
material damping," Experimental Mechanics, vol. 17, no. 6, pp. 297-302, 1977.

[7] N. Srikanth and M. Gupta, "Damping Characterization of Monolithic and Reinforced Magnesium Using an Integrated Suspended Beam Method and a New Circle-Fit Approach," physica status solidi (a), vol. 193, no. 2, pp. 236-245, 2002.

[8] JM Berthelot and Y Sefrani, "Damping analysis of unidirectional glass and Kevlar fibre composites," Composites Science and Technology, vol. 64, no. 9, pp. 12611278, July 2004.

[9] Roger M. Crane and John W. Gillespie, "Characterization of the vibration damping loss factor of glass and graphite fiber composites," Composites Science and Technology, vol. 40, no. 4, pp. 355-375, 1991.

[10] Wilfred E. Baker, William E. Woolam, and Dana Young, "Air and internal damping of thin cantilever beams," International Journal of Mechanical Sciences, vol. 9, no. 11, pp. 743-766, 1967.

[11] Shafi Ullah Khan, Chi Yin Li, Naveed A. Siddiqui, and Jang-Kyo Kim, "Vibration damping characteristics of carbon fiber-reinforced composites containing multiwalled carbon nanotubes," Composites Science and Technology, vol. 71, no. 12, pp. 1486-1494, Aug 2011.

[12] R. D. Adams and M. A. O., "Measurement of the damping capacity and dynamic modulus of high-damping metals under direct cyclic stresses," Journal of Physics D: Applied Physics, vol. 5, no. 7, pp. 1274-1283, 1972.

[13] JP Talbot and J Woodhouse, "The vibration damping of laminated plates," Composites Part A - Applied Science and Manufacturing, vol. 28, no. 12, pp. 1007$1012,1997$.

[14] V Kostopoulos and DT Korontzis, "A new method for the determination of 
viscoelastic properties of composite laminates: a mixed analytical-experimental approach," Composites Science and Technology, vol. 63, no. 10, pp. 1441-1452, Aug 2003.

[15] A. Srikantha Phani and J. Woodhouse, "Experimental identification of viscous damping in linear vibration," Journal of Sound and Vibration, vol. 319, no. 3-5, pp. 832-849, Jan 2009.

[16] M.R. Maheri and R. D. Adams, "Finite-element prediction of modal resposne of damped layered composite panels," Composites Science and Technology, vol. 55, no. 1 , pp. 13-23, 1995.

[17] J DeVisscher, H Sol, WP DeWilde, and J Vantomme, "Identification of the damping properties of orthotropic composite materials using a mixed numerical experimental method," Applied Composie Materials, vol. 4, no. 1, pp. 13-33, 1997.

[18] C.W. Bert, "Material damping - introductory review of mathematical models, measures and experimental techniques," Journal of sound and vibration, vol. 29, no. 2, pp. 129-153, 1973.

[19] J. Woodhouse, "Linear damping models for structural vibration," Journal of Sound and Vibration, vol. 215, no. 3, pp. 547-569, 1998.

[20] S. H. Crandall, "On scaling laws for material damping," National Aeronautics and Space Administration, NASA Technical report 1962.

[21] P. J. Torvik, "Material and slip damping," in Harris' Shock and Vibraiton Handbook.: McGraw-Hill, 2010, ch. 35, pp. 932-960.

[22] B. J. Lazan and L. E. Goodman, Material and Inteface Damping, Cyril M. Harris and Charles E. Crede, Eds.: McGraw-Hill, 1976, vol. Chap. 36 of Shock and Vibration Handbook. 
[23] D. J. Ewins, Modal testing: Theory and Practice.: Research Studies Press Ltd., 1984.

[24] M.R. Maheri and R.D. Adams, "Modal vibration damping of anisotropic FRP laminates using the Rayleigh-Ritz energy minimization scheme," Journal of Sound and Vibration, vol. 259, no. 1, pp. 17-29, Jan 2003.

[25] ASTM Standard E756-05, Standard test method for measuring vibration damping Damping properties of materials, 2010.

[26] R.F. Gibson, "Modal vibration response measurements for characterization of composite materials and structures," Composites Science and Technology, vol. 60, no. 15 , pp. 2769-2780, 2000.

[27] A.M.R. Ribeiro, J.M.M. Silva, N.M.M. Maia, and L. Reis, "Free Vibration Response Using The Constant Hysteretic Damping Model," in The 11th International Conference on Vibration Engineering, Timisoara, Romania, September 27-30 2005.

[28] B. Harras, R. Benamar, and R.G. White, "Geometrically non-linear free vibration of fully clamped symmetrically laminated rectangular composite plates," Journal of Sound and Vibration, vol. 251, no. 4, pp. 579-619, Apr 2002.

[29] J.M. Lee and K.G. McConell, "Experimental Cross Verification of Damping in Three Metals," Experimental Mechanics, vol. 15, pp. 347-353, 1975.

[30] Joachim Vanwalleghem, Ives De Baere, Mia Loccufier, and Wim Van Paepegem, "Practical aspects in measuring vibration damping of materials," in Proceedings of the 15th International conference on experimental mechanics, Porto, 2012.

[31] J. Vanwalleghem, "Study of the damping and vibration behaviour of flax-carbon composite bicycle racing frames," Ghent University, Master's thesis 2009-2010. 
[32] D.T. Korontzis, L. Vellios, and V. Kostopoulos, "On the viscoelastic response of composite laminates," Mechanics of Time-Dependent Materials, vol. 4, no. 4, pp. 381-405, 2000.

[33] M. Colakoglu, "Effect of temperature on frequency and damping properties of polymer matrix composites," Advanced Composite Materials, vol. 17, no. 2, pp. 111-124, 2008. 
List of figure captions:

Figure 1: Resonant mode shapes from square steel plate with dimensions $300 \times 300 \times 2$ mm. a) First resonant mode shape; b) Second resonant mode shape

Figure 2: Free vibration decay from accelerometer mounted at anti-nodal position of first resonant frequency. a) Attenuation acceleration signal as function of time after loudspeaker excitation is switched off; b) Power spectral density from decaying acceleration signal in Figure 2.a.

Figure 3: Influence of sensor wire mounting on decaying vibration response: amplitude normalized envelope curve of response signal

Figure 4: Strain level and hot spot locations for first mode shape. a) Shear strain E12; b) Longitudinal strain E11

Figure 5: Test configuration through contactless excitation and response measurement Figure 6: Mismatch between original response signal and mathematical curve from the viscous damping model (Eq. (1))

Figure 7: Visualization of nonlinear damping

Figure 8: Vibration response with fixed strain gauge lead wire for shear strain measurement. a) velocity response; b) strain response

Figure 9: Vibration response with loose strain gauge lead wire for shear strain measurement. a) velocity response; b) strain response

Figure 10: Vibration response with loose strain gauge lead wire for longitudenal strain measurement. a) velocity response; b) strain response

Figure 11: Damping ratio as function of sample size, sample size given as first resonant frequency 
Figure 12: Damping as function of specimen response amplitude for three specimen sizes. a) First mode shape; b) Second mode shape

List of Table captions:

Table 1: Mechanical properties of test specimens

Table 2: Nonlinear damping ratio: damping value depends on response amplitude

Table 3: Overview of damping values $\zeta\left(\cdot 10^{-5}\right)$ with different response measuring methods for the first resonance mode. A: Doppler vibrometer; B: shear strain with fixed wire; C: shear strain with loose wire; D: longitudinal strain with loos wire; E: accelerometer with fixed wire; F: accelerometer with loose wire

Table 4: Damping values at different sample-loudspeaker distances for the largest sample $300 \times 300 \times 2 \mathrm{~mm}$

Table 5: Relative air damping contribution for the largest sample 300x300x2 mm

Table 6: Relative air damping contribution for amplitude range $2.5-1.25 \mathrm{~mm} / \mathrm{s}$

Table 7: Error due to specimen suspension at anti-nodal position of first mode shape for different suspension lengths and specimen size

Table 8: Error due to specimen suspension at anti-nodal position of second mode shape for different suspension lengths and specimen size

Table 9: Comparison of damping ratio for different steel (alloy) materials 


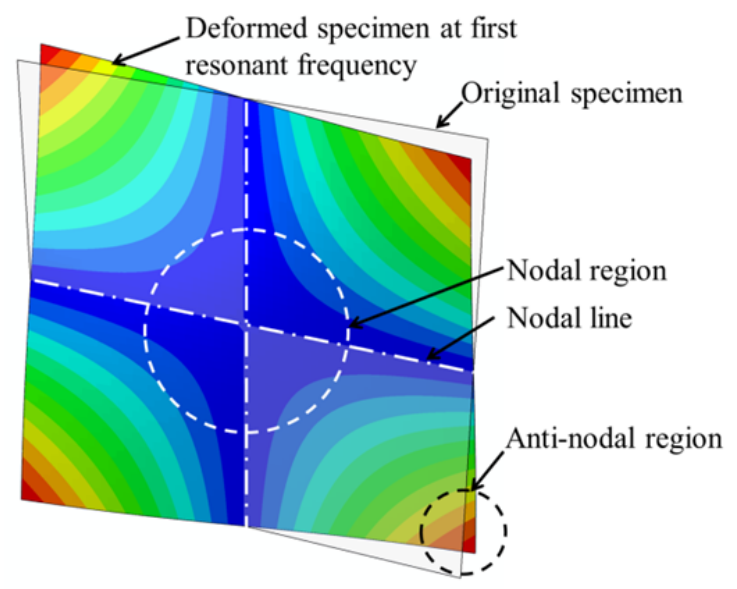

a)

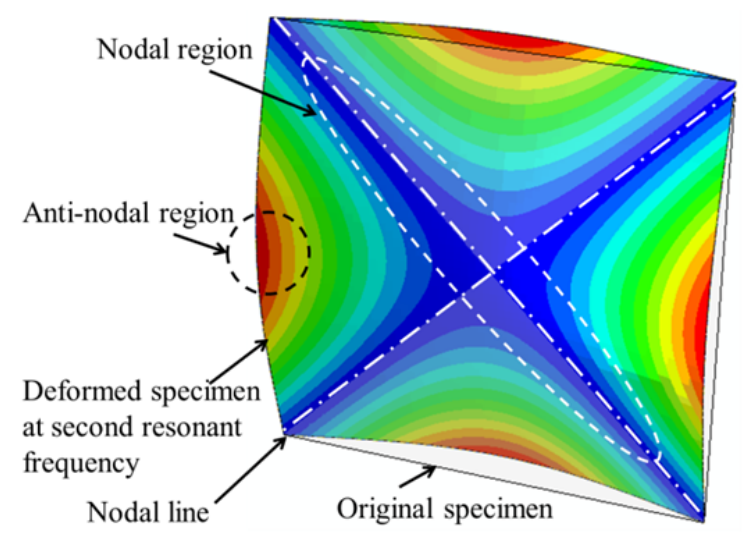

b)

Figure 1: Resonant mode shapes from square steel plate with dimensions 300x300x2 mm. a) First resonant mode shape; b) Second resonant mode shape

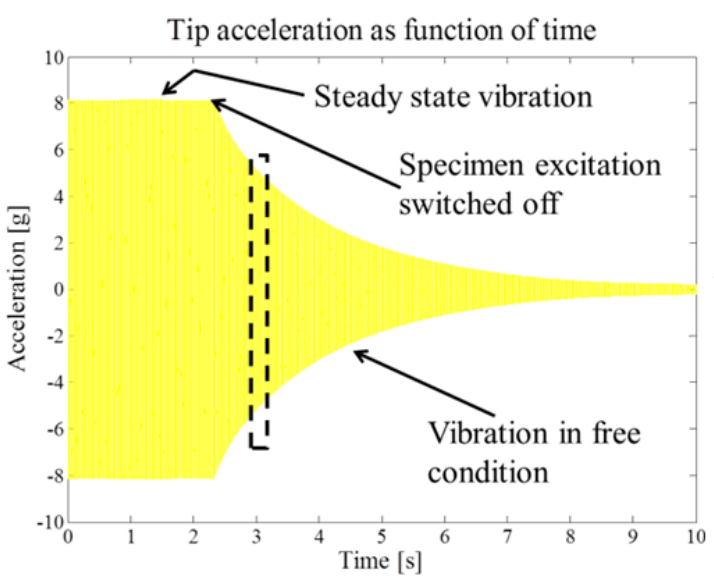

a)

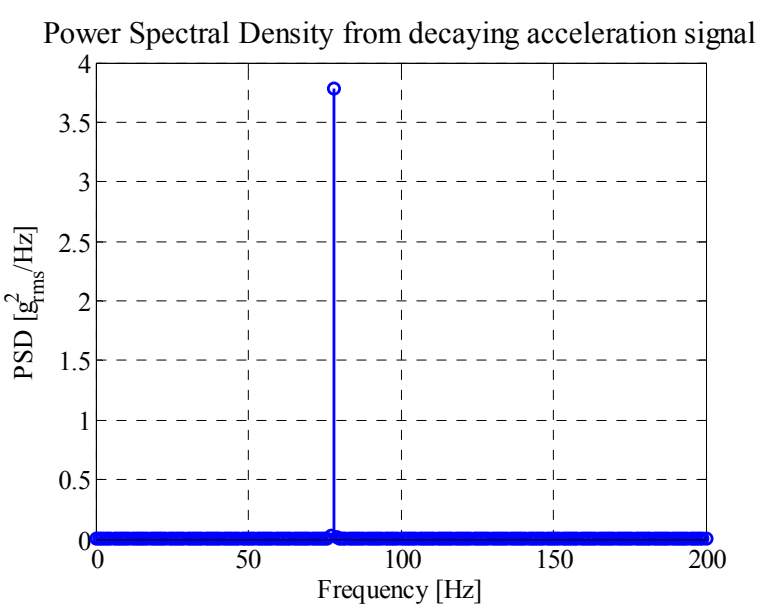

b)

Figure 2: Free vibration decay from accelerometer mounted at anti-nodal position of first resonant frequency. a) Attenuation acceleration signal as function of time after loudspeaker excitation is switched off; b) Power spectral density from decaying acceleration signal in Figure 2.a. 


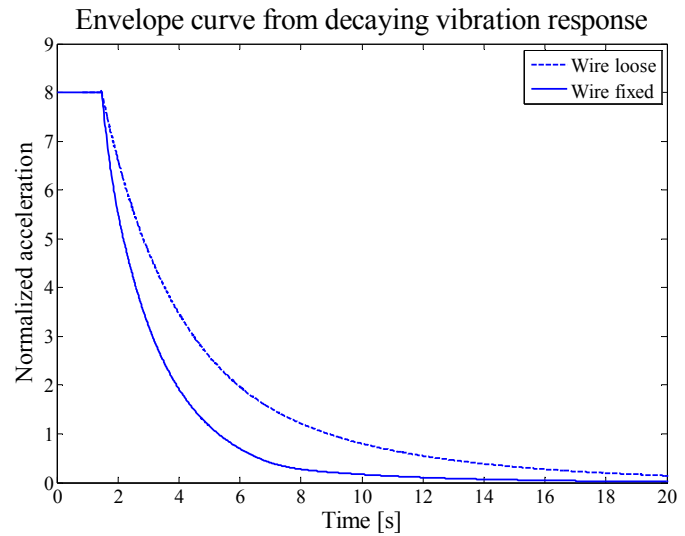

Figure 3: Influence of sensor wire mounting on decaying vibration response: amplitude normalized envelope curve of response signal
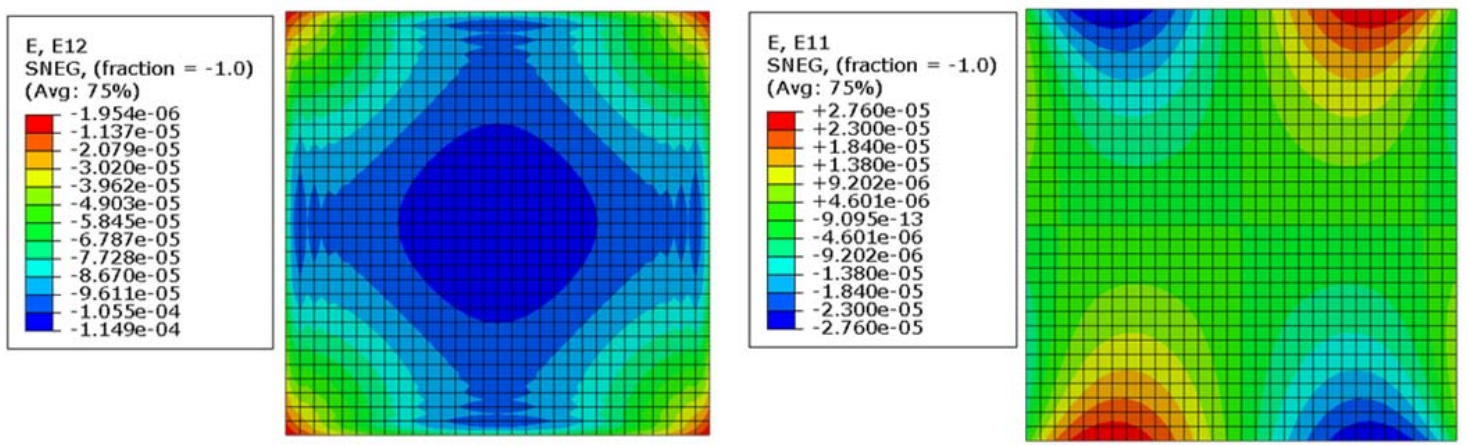

Figure 4: Strain level and hot spot locations for first mode shape. a) Shear strain E12; b)

Longitudinal strain E11 


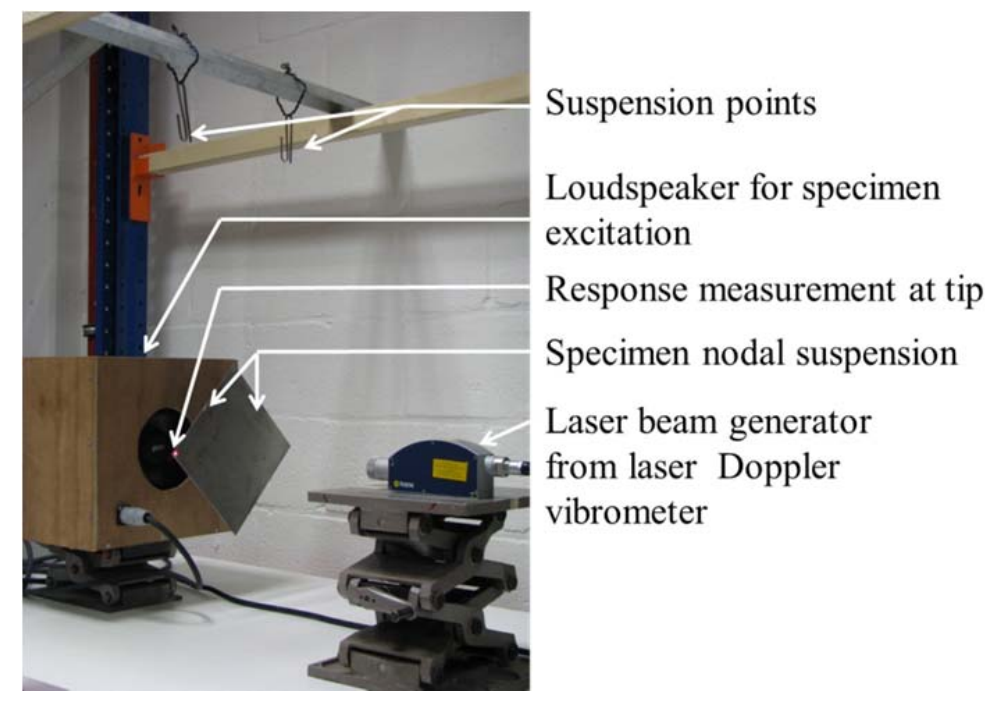

Figure 5: Test configuration through contactless excitation and response measurement

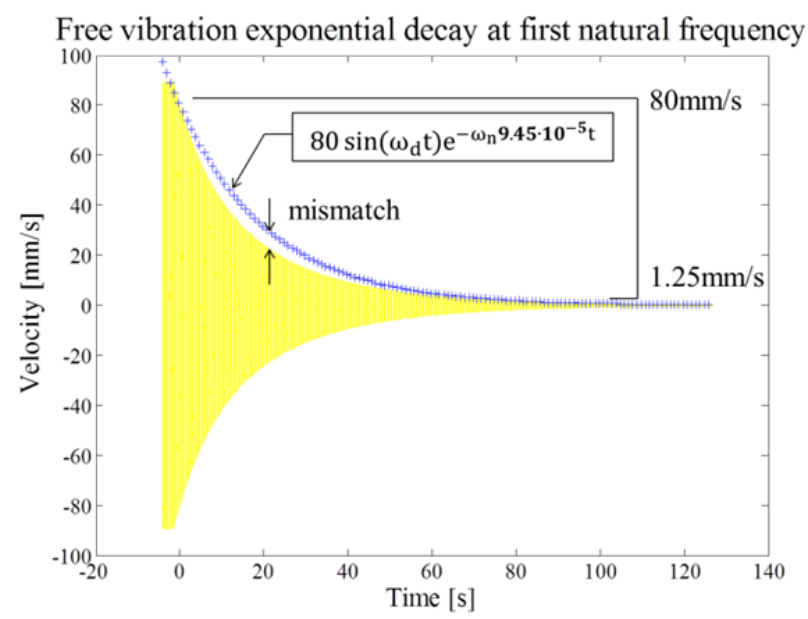

Figure 6: Mismatch between original response signal and mathematical curve from the viscous damping model (Eq. (1))

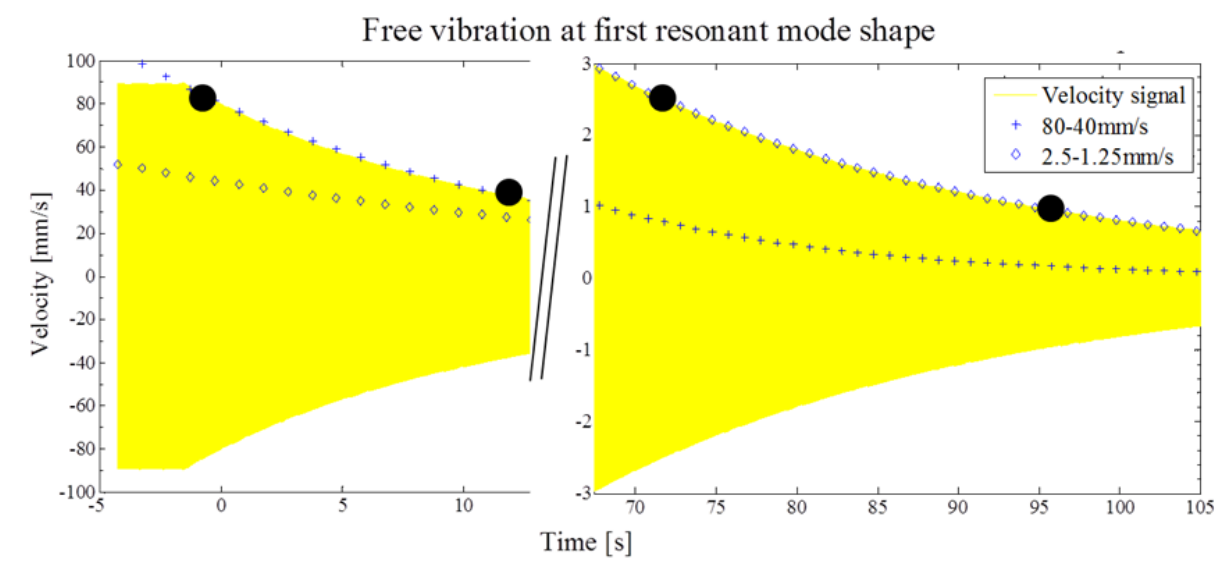

Figure 7: Visualization of nonlinear damping 

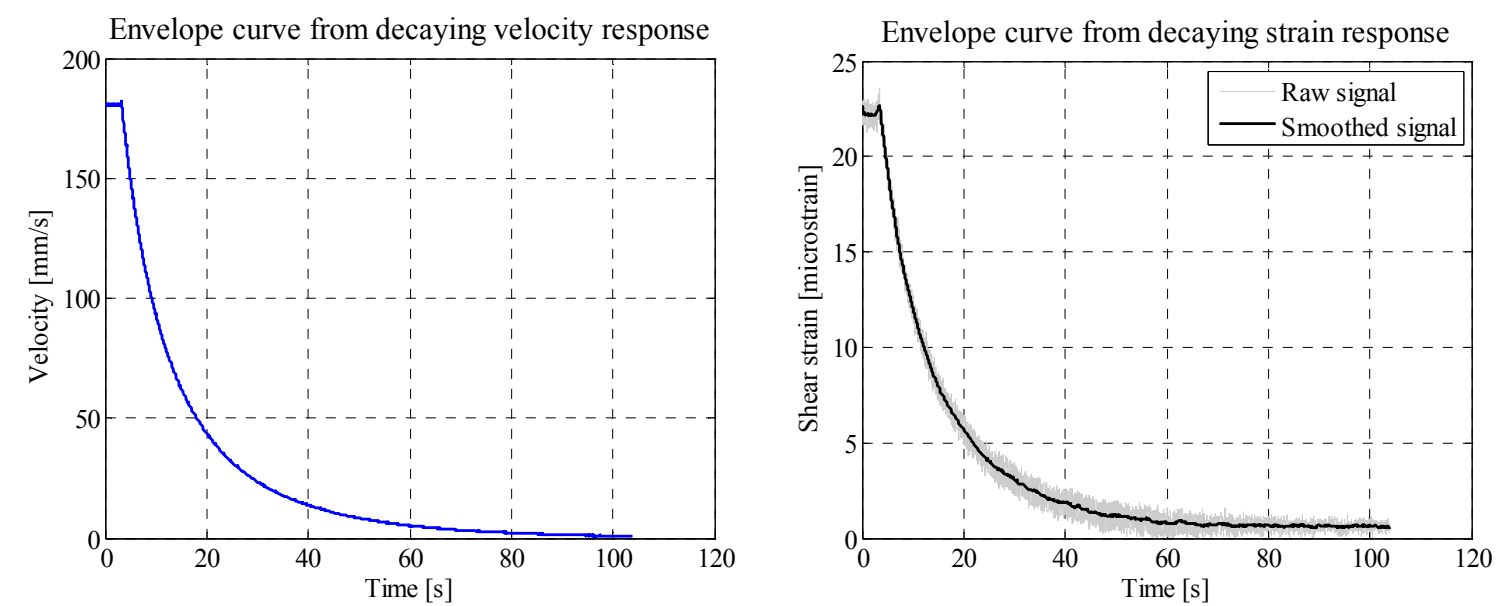

Figure 8: Vibration response with fixed strain gauge lead wire for shear strain measurement. a) velocity response; b) strain response
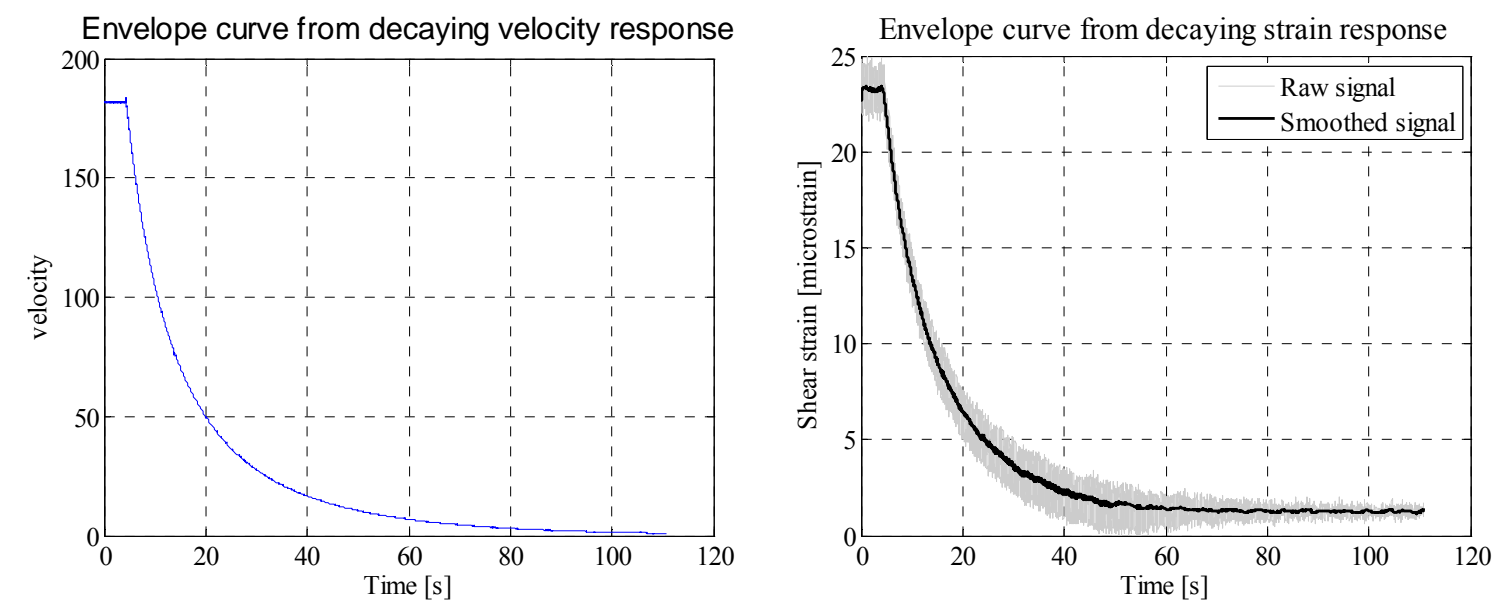

Figure 9: Vibration response with loose strain gauge lead wire for shear strain measurement. a) velocity response; b) strain response
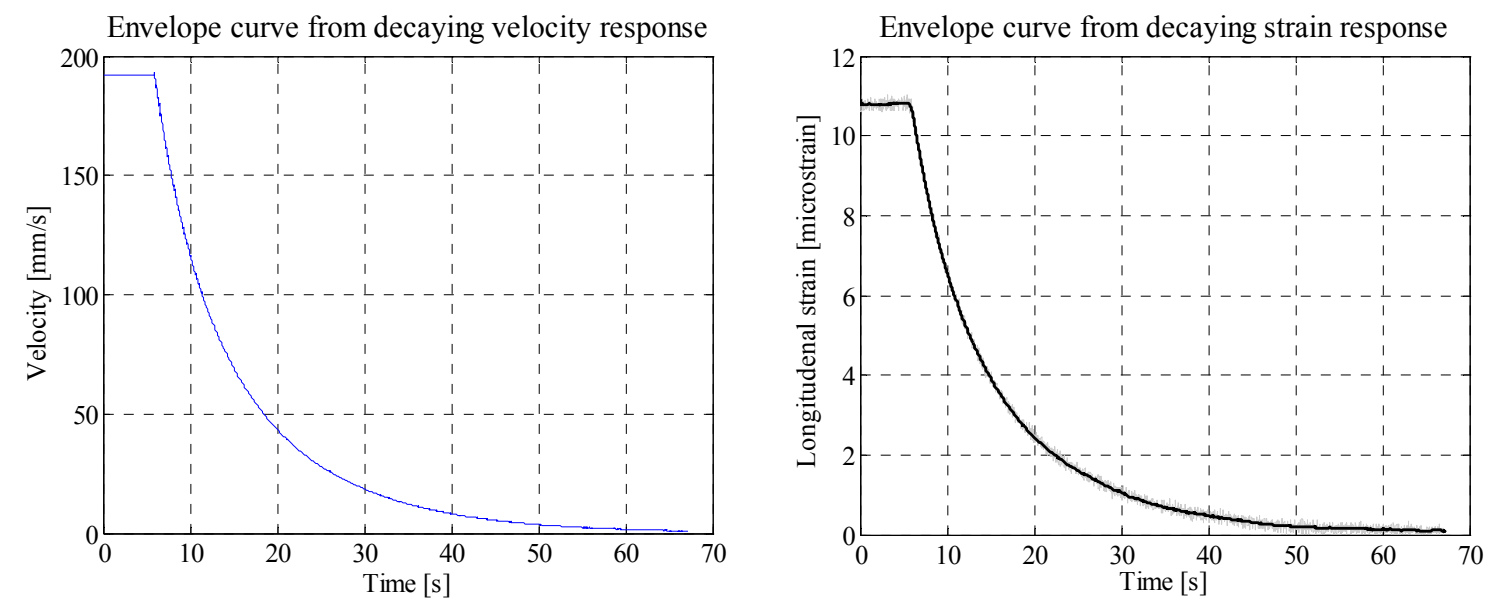

Figure 10: Vibration response with loose strain gauge lead wire for longitudinal strain measurement. a) velocity response; b) strain response 


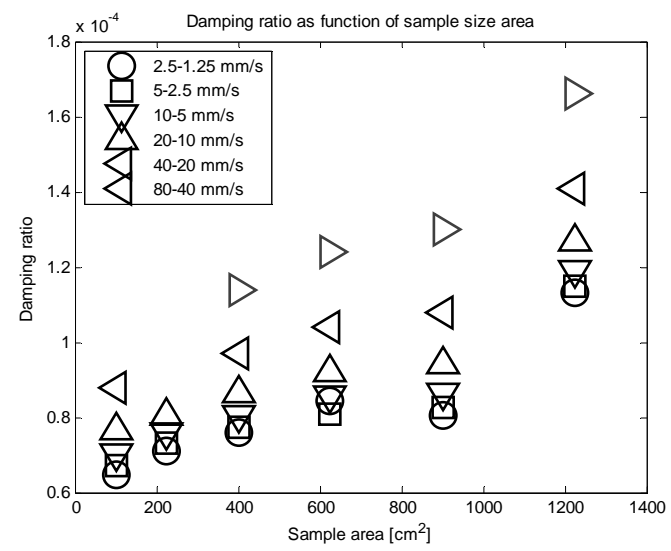

Figure 11: Damping ratio as function of sample size, sample size given as first resonant frequency
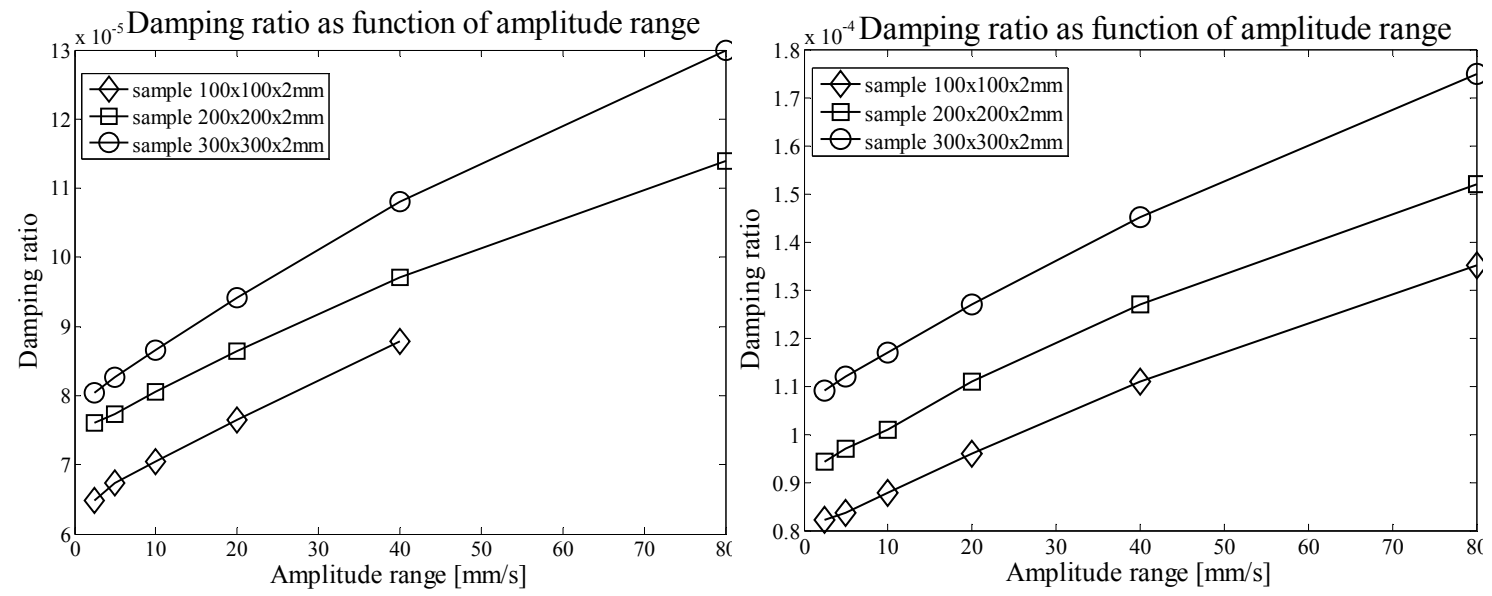

a)

b)

Figure 12: Damping as function of specimen response amplitude for three specimen sizes. a) First mode shape; b) Second mode shape 